Document downloaded from:

http://hdl.handle.net/10251/99609

This paper must be cited as:

Lopez Orti, JA.; Marco Castillo, FJ.; Martínez Uso, MJ. (2014). Study of errors in the integration of the two-body problem using generalized Sundman's anomalies. SEMA SIMAI Springer Series. 4:105-112. doi:10.1007/978-3-319-06953-1_11

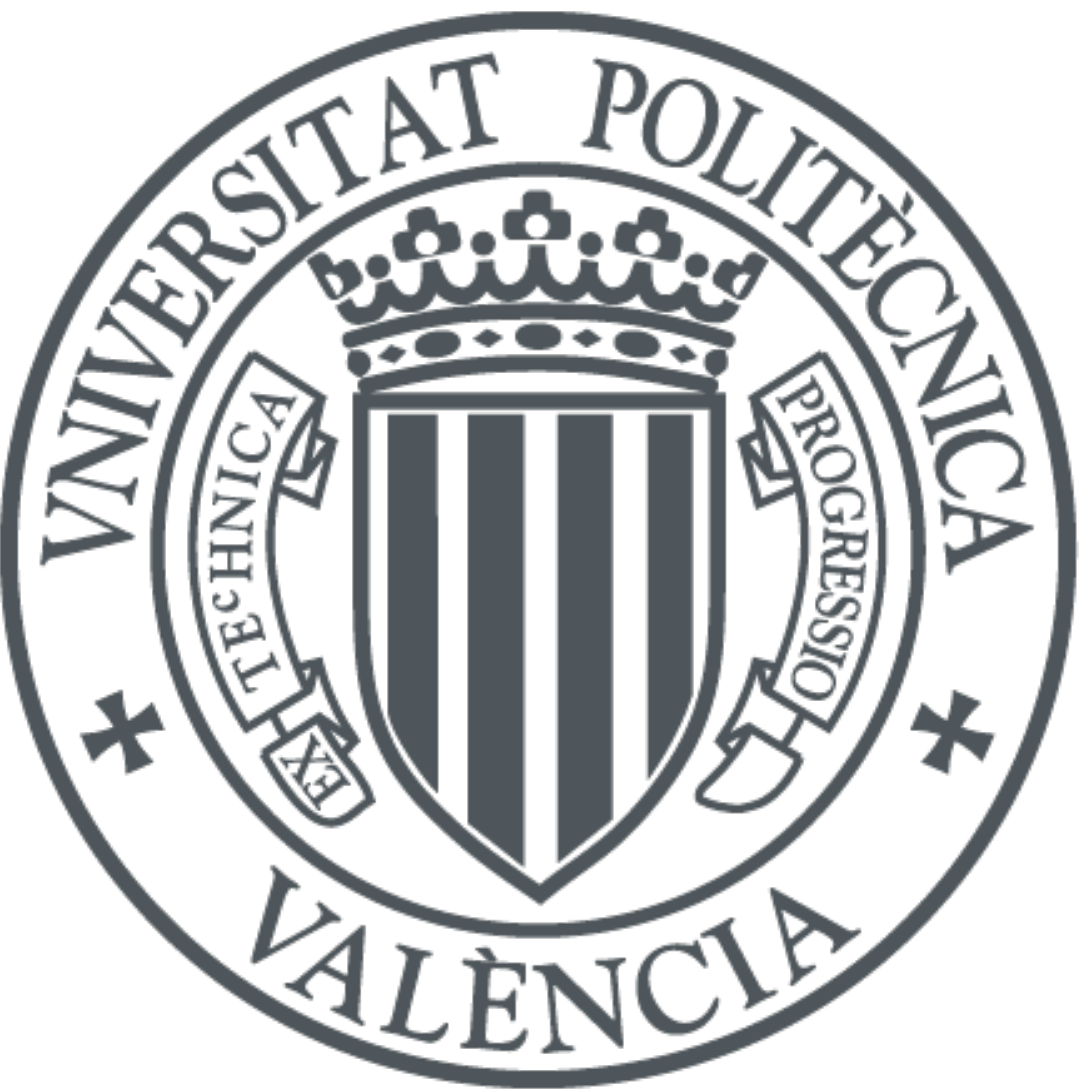

The final publication is available at

https://doi.org/10.1007/978-3-319-06953-1_11

Copyright Springer

Additional Information 


\title{
Study of Errors in the Integration of the Two Body Problem Using Generalized Sundman's Anomalies
}

\author{
José Antonio López Ortí, Francisco José Marco Castillo, and María José \\ Martínez Usó
}

\begin{abstract}
As is well known, the numerical integration of the two body problem with constant step presents problems depending on the type of coordinates chosen. It is usual that errors in Runge-Lenz's vector cause an artificial and secular precession of the periaster although the form remains symplectic, theoretically, even when using symplectic methods. Provided that it is impossible to preserve the exact form and all the constants of the problem using a numerical method, a possible option is to make a change in the variable of integration, enabling the errors in the position of the periaster and in the speed in the apoaster to be minimized for any eccentricity value between 0 and 1 .

The present work considers this casuistry. We provide the errors in norm infinite, of different quantities such as the Energy, the module of the Angular Moment vector and the components of Runge-Lenz's vector, for a large enough number of orbital revolutions.
\end{abstract}

\section{Introduction}

One of the principal problems present in spatial mechanics is the integration of the equations of motion of an artificial satellite in orbit around the Earth. This motion can be approached in a geocentric system of coordinates by means of the equations [2]

$$
\frac{d^{2} \mathbf{r}}{d x^{2}}=-\frac{G m}{r^{3}} \mathbf{r}-\nabla U+\mathbf{F}
$$

\footnotetext{
J.A. López Ortí $(\bowtie) \cdot$ F.J. Marco Castillo

Departamento de Matemáticas, Universidad Jaume I de Castellón, Av Sos Baynat s/n, 12071

Castellón, Spain

e-mail: lopez@mat.uji.es; marco@mat.uji.es

M.J. Martínez Usó

Departamento de Matemática Aplicada, Universidad Politécnica de Valencia, Camino Vera s/n, Valencia, Spain

e-mail: mjmartin@mat.upv.es
} 
where $\mathbf{r}$ is the vector of geocentric position of the satellite, $G$ is the constant of universal gravitation, $m$ is the terrestrial mass, $U$ is the generating potential of the conservative perturbing forces, such as the luni-solar and other planets attraction as well as the forces due to the not sphericity of the Earth and $\mathbf{F}$ represents the non conservative perturbing forces such as the friction with the higher layers of the atmosphere, etc.

The integration of the previous problem can be carried out by means of analytical techniques from Gauss's planetary equations [8] or using numerical techniques, with the choice of an appropriate numerical method together with a convenient step. The perturbing forces acting on a satellite are usually small, so a common procedure in the construction of integrators adapted for this problem is the development of efficient integrators for the two body problem (this is the not disturbed problem) and then using them for the resolution of the general problem. The study here presented is focused on the elliptic motion. In this case, the two major problems appear when the eccentricity is high. First, the temporary distribution of points on the orbit is very unequal depending on the region. Second, the orbit has zones with very different curvatures. To settle these problems and obtain an acceptable precision, we can use several techniques [14] such as the choice of a very small uniform step, a variable step, and finally a change of the temporal variable so that a better temporal distribution of positions is obtained on the orbit near the perigee where the speed of the satellite is faster without reducing excessively the concentration of positions in the perigee, where the curvature, as well as in the perigee, is maximum. The problem of reparameterization of the temporal variable has been studied by several author $[2,4,7,10,11]$ using several kinds of anomalies.

In this work we follow this third way, we study especially a family of transformations derived from $d t=K_{\alpha} r^{\alpha} d \tau$ [7], called Sundman's generalized transformations.

In this section we briefly explain the terminology associated to the two body problem. A more detailed version can be seen in [1,13]. The two body problem is a classic celestial mechanics problem regarding to the problem of the motion of two punctual bodies under the action of their gravitational forces. One of the most usual ways of studying this problem is by means of the study of the relative motion: the motion of a body, generally the one of smaller mass, called secondary, with respect to that of higher mass, called primarily. If $\mathbf{r}$ is the vector of position of the secondary with respect the primary, the motion follows the equation

$$
\ddot{\mathbf{r}}=-\mu \frac{\mathbf{r}}{r^{3}}, \mu=G\left(m+m^{\prime}\right), \mathbf{r}(0)=\mathbf{r}_{0}, \dot{\mathbf{r}}(0)=\mathbf{v}_{0}
$$

It is known that the two body problem satisfies Kepler's laws. The orbits of the secondary with respect to the primary are conical, with the primary in the principal focus, the area swept by the radius vector that links the primary with the secondary is proportional to the time; and the reason between the cube of the major semiaxis and the square of the period is constant for an elliptical orbit. 
In the two body problem appear several important magnitudes such as the integral of the areas $\mathbf{C}=\mathbf{r} \times \mathbf{v}$ whose meaning is the double of the areolar speed. On the other hand, we have also that the vector $A$, called the Laplace-Runge-Lenz's vector defined as

$$
\mathbf{A}=\mathbf{v} \times \mathbf{C}-\mu \frac{\mathbf{r}}{r}
$$

is constant. It is usual to represent the vector as $\mathbf{A}=\mu \mathbf{e}$.

The equation of the relative orbit is obtained computing the scalar multiplication of $\mathbf{r}$ and $\mu \mathbf{e}$ providing

$$
r=\frac{p}{1+e \cos V}
$$

where $p=\frac{C^{2}}{\mu}$ is the parameter of the conic, $e$ the eccentricity and $V$ the angle between $\mathbf{A}$ and $\mathbf{r}$, known as true anomaly. This angle is measured from $\mathbf{A}$. The $\mathbf{A}$ vector determines the direction of the periaster and its norm is directly related to the eccentricity $e$.

In addition, $h$ is constant too. $h$ is the integral of the energy and its value is

$$
h=\frac{1}{2} v^{2}-\frac{\mu}{r}
$$

where $v^{2}=\mathbf{v} \cdot \mathbf{v}$.

In the case of the elliptical motion $(0 \leq e<1)$ the value of the parameter is given by $p=a\left(1-e^{2}\right)$ and the period $P$ by $P=\frac{2 \pi a^{2} \sqrt{1-e^{2}}}{C}$, where $a$ it is the major semi axis of the ellipse. In this case, we also define the mean motion $n$ as $n=\frac{2 \pi}{P}$ and the mean anomaly as $M=n\left(t-T_{0}\right)$ where $T_{0}$ is the epoch of the closest approach.

Finally, in the elliptical motion it is also of great interest the so called eccentric anomaly $E$ related to the mean anomaly $M$ through Kepler's equation $E-e \sin E=M$.

If the orbital system of coordinates $(x, y)$ is considered, with $O$ placed in the primary focus, $O X$ in the direction of the periaster and $O Y$ perpendicular to $O X$ so that the motion takes place in direct sense, it turns out that $r=a(1-e \cos E)$, $x=r \cos V=a(\cos E-e), y=r \sin V=a \sqrt{1-e^{2}} \sin E$.

In Sect. 2 we briefly study the generalized family of Sundman's anomalies, that we use as temporal variables in the numerical integration of the two body problem.

In Sect. 3 we compute the numerical integration of different examples of the simple two body problem along 100,000 revolutions. We study the effect in the integral of the areas and the energy of the $\alpha$ value for different eccentricities. We also study the dependence of $\alpha$ on the eccentricity and the numerical precession considering Runge-Lenz's vector along long periods of time.

In section "Conclusions" we give the main conclusions of the work. 


\section{Study of the Family of Sundman Generalized Anomalies}

In the year 1912 Sundman [12], introduced the change of temporary variable $d t=C r d \tau$ in order to regularize the problem of three bodies. Later Nacozy $[6,11]$ extended this transformation to a more general $d t=C_{\alpha} r^{\alpha} d \tau$, such family of transformations includes the mean, eccentric and true anomalies for values of $\alpha=$ $0,1,2$ and appropriate values of $C_{\alpha}$ [9]. From the above mentioned family Lopez [9] introduces the concept of Sundman's generalized anomaly $\Psi_{\alpha}$ as a function $\Psi_{\alpha}(M)$ so that

- $d M=K_{\alpha}(e) r^{\alpha} d \tau, K_{\alpha}(e)=n C_{\alpha}$ being $n$ is the mean motion.

- $\Psi_{\alpha}(\pi)=\pi, \Psi_{\alpha}(2 \pi)=2 \pi$.

- $\Psi_{\alpha}(M+2 \pi)=\Psi_{\alpha}(M), \Psi_{\alpha}(-M)=-\Psi_{\alpha}(M)$.

To this aim, it is sufficient that

$$
K_{\alpha}(e)=\frac{1}{2 \pi} \int_{0}^{2 \pi}(1-e \cos E)^{1-\alpha} d E
$$

whose value is given by [9]

$$
K_{\alpha}(e)=a^{-\alpha}\left\{(1-e)^{1-\alpha} F\left(\frac{1}{2}, \alpha-1,1 ; \frac{2 e}{e-1}\right)+(1+e)^{1-\alpha} F\left(\frac{1}{2}, \alpha-1,1 ; \frac{2 e}{1+e}\right)\right\}
$$

where $F(a, b, c ; z)$ is the hypergeometrical function.

The function $\Psi_{\alpha}-M$ can be developed as Fourier series depending on $M$ and as Fourier series of $\Psi_{\alpha}$ [9], where the development of $\frac{1}{r}, r \sin V$ y $r \cos V$ is also obtained as Fourier series depending on $\Psi_{\alpha}$. So, a set of developments sufficient for the analytical treatment of the problem is provided.

With regard to the concerned numerical methods, the differential equations of motion depend on the $t$ variable; in this way, we have

$$
\frac{d}{d t}=n \frac{d}{d M}=\frac{n}{K_{\alpha}(e)} r^{-\alpha} \frac{d}{d \Psi_{\alpha}} .
$$

Thus, the equations of motion of the two body problem in the orbital coordinates $(x, y)$ are

$$
\begin{array}{ll}
\frac{d x}{d \Psi_{\alpha}}=\frac{K_{\alpha}(e)}{n} r^{\alpha} v_{x}, & \frac{d v_{x}}{d \Psi_{\alpha}}=-\frac{K_{\alpha}(e)}{n} r^{\alpha} G M \frac{x}{r^{3}} \\
\frac{d y}{d \Psi_{\alpha}}=\frac{K_{\alpha}(e)}{n} r^{\alpha} v_{y}, & \frac{d v_{y}}{d \Psi_{\alpha}}=-\frac{K_{\alpha}(e)}{n} r^{\alpha} G M \frac{y}{r^{3}} .
\end{array}
$$


The use of an appropriate value for the $\alpha$ parameter improves the efficiency of the integration in the two body problem. The optimal value for $\alpha$ for each value of eccentricity can be approached by

$$
\alpha(e)=1.5541 e^{4}-1.94142 e^{3}+0.582338 e^{2}+0.252954 e+1.54422 .
$$

The robustness of these value has been tested using a Runge-Kutta of eight order [3] and Gragg-Bulirsch-Stoer integrators [5].

\section{Numerical Results}

In the present section we study the motion on the orbital plane of a fictitious satellite with the same orbital elements that the old HEOSII satellite except for the eccentricity, that is modified to simulate different cases. As one would expect, low values of the eccentricity do not provide significantly different results. To test the efficiency of the integrators for higher values of the eccentricity we consider a high value $e=0.5$ and an extreme value $e=0.95$.

First we carry out the integration of a satellite with a high eccentricity $e=0.5$, considering 10,000 orbits, using a classic Runge-Kutta method of fourth order with 1,000 uniform steps. Firstly we employ the mean anomaly $\alpha=0$ and secondly the Nacozy's intermediate anomaly $\alpha=1.5$. In this last case, the results are improved. Figure 1 shows the magnitude of the variations in the quantities $C$, $H, e, \omega$, that are constants at the perigee in the analytical solution of two body problem, depending on the used anomaly. In each subfigure the OX axis represents the number of revolutions and the $O Y$ axis the value of the quantities $C, H, e$ is the eccentricity and $\omega$ is in radians. For the initial epoch $t=0 C=188,109.144$ and $H=-1.68376245$

The integration is repeated for a case with extreme eccentricity $e=0.95$. In this case, the use of the mean anomaly as variable of integration provides absolutely inadmissible results. We obtain considerably improved results in the case $\alpha=1.9$, shown in Fig. 2. For $t=0 C=67,823.519$.

The long time error in the quantities of the energy $H$, the areas integral $C$, the eccentricity $e(t)$ and the numerical precession of the perigee $\omega$ can be improved using an appropriate value of $\alpha$. If $e=0.5$ and $e=0.95$ the dependence of the results on the value of the chosen parameter $\alpha$ is evident. In order to test the robustness of the method, these results have been compared with the ones obtained using a Bulirsch-Stoer method. In both cases the results are similar. 

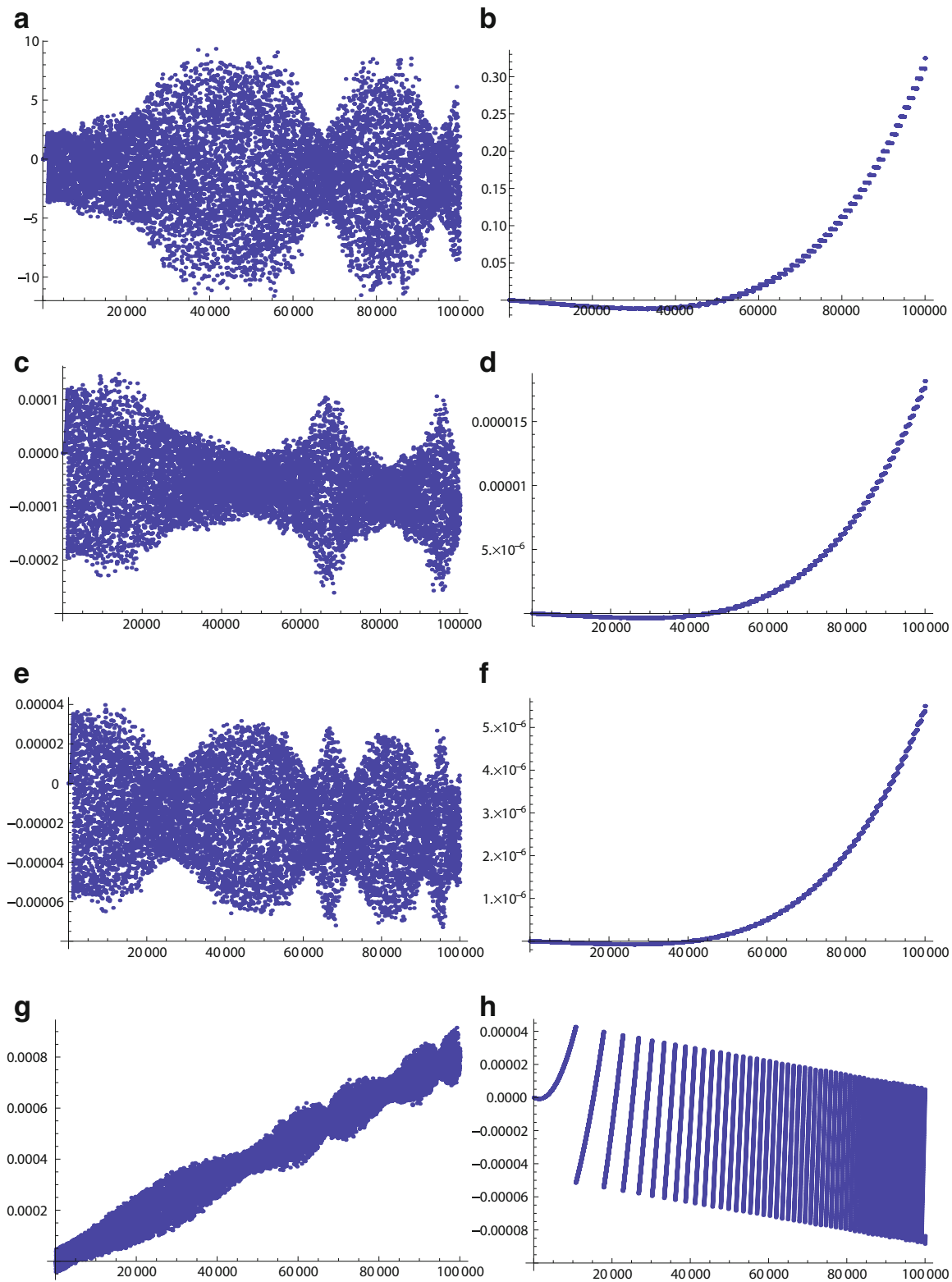

Fig. 1 Time evolution of $\triangle C, \triangle H, \Delta e$ and $\triangle \omega$. (a) $\triangle C: e=0.5 \alpha=0.0$. (b) $\triangle C$ : $\mathrm{e}=0.5$. $\alpha=1.5$. (c) $\Delta H: e=0.5 \alpha=0.0$. (d) $\Delta H: e=0.95 \alpha=1.9$. (e) $\Delta e(t): e=$ $0.5 \alpha=0.0$. (f) $\Delta e(t): e=0.5 \alpha=1.5$. (g) $\Delta \omega: e=0.5 \alpha=0.0$. (h) $\Delta \omega: e=0.5 \alpha=$ 1.5 
a

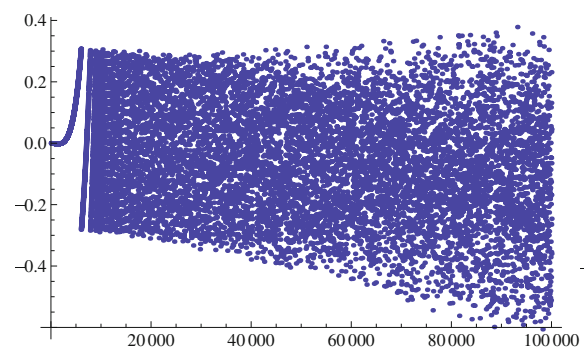

C

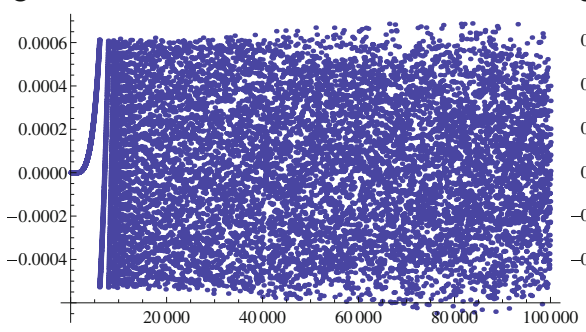

b

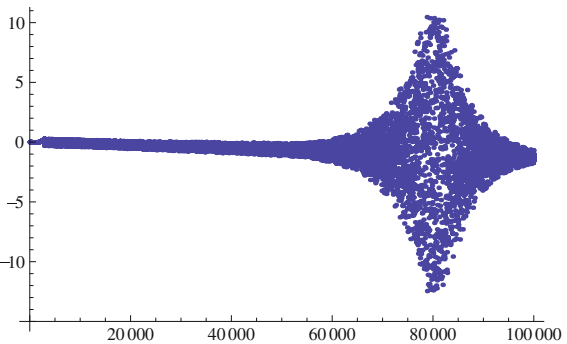

d

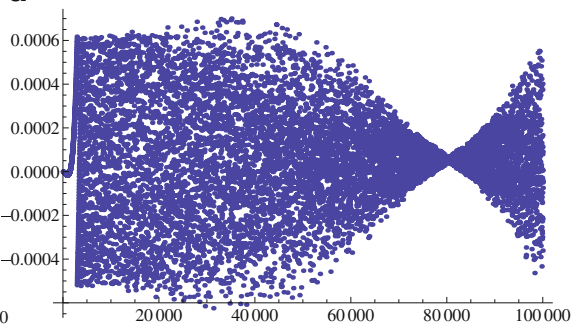

f
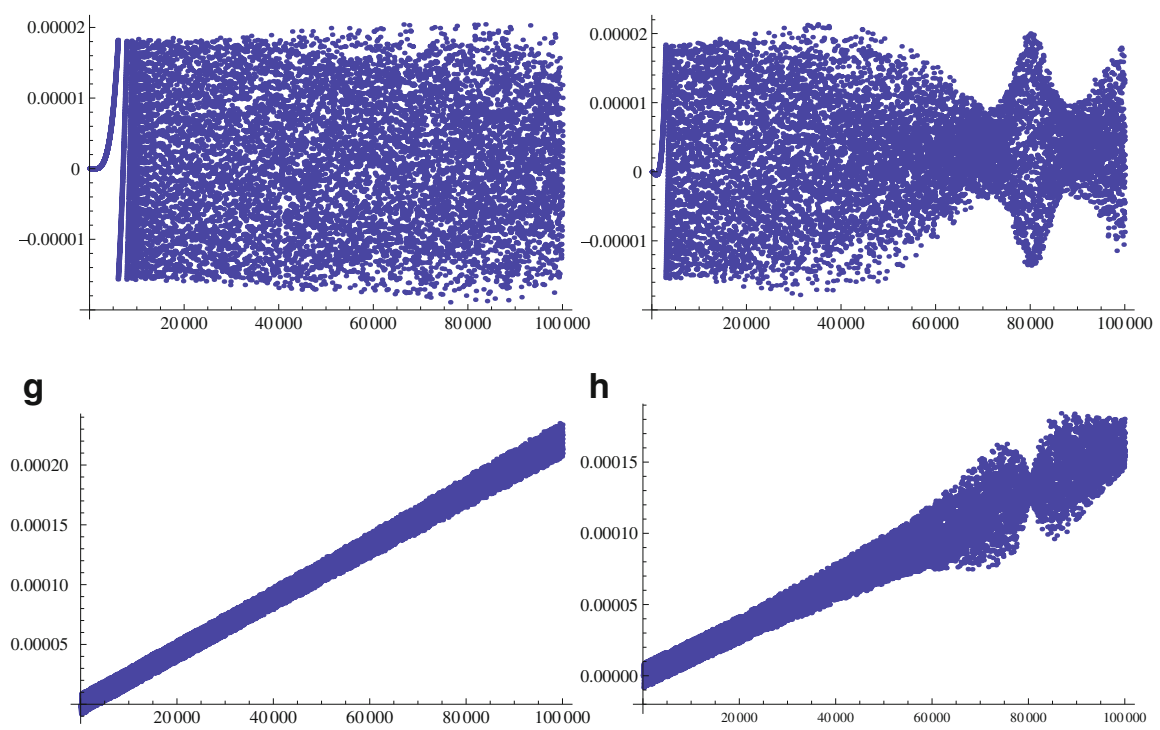

h

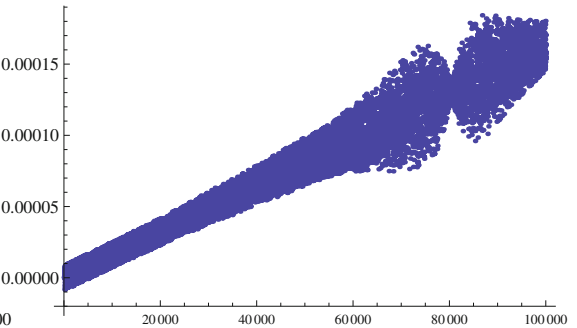

Fig. 2 Time evolution of $C, H, e$ and $\omega$. (a) $\triangle C: e=0.95 \alpha=1.5$. (b) $\triangle C: e=0.95 \alpha=$ 1.9. (c) $\triangle H: e=0.95 \alpha=1.5$. (d) $\triangle H: e=0.95 \alpha=1.9$. (e) $\Delta e(t): e=0.95 \alpha=1.5$. (f) $\Delta e(t): e=0.95 \alpha=1.9$. (g) $\Delta \omega: e=0.95 \alpha=1.5$. (h) $\Delta \omega: e=0.95 \alpha=1.9$ 


\section{Conclusions}

In this work it is remarked that it is of great importance the temporary variable chosen in the numerical integration of the orbital motion. The use of an appropriate anomaly from the family of Sundman's generalized anomalies improves the preservation along long temporal periods of quantities that must remain invariant in the two body problem. The first integrals given by the constant of the areas, the energy, the direction of apoaster and the LaplaceRunge-Lenz's vector, which determines the value of the eccentricity, are slightly sensitive to the value of $\alpha$ for small eccentricities. When the value of the eccentricity increases, the conservation of these quantities is a much more delicate problem. For extreme values of the eccentricity $e=0.95$ the results obtained for low values of $\alpha$ are inadmissible. In these cases, the most adequate values for $\alpha$ are between 1.5 and 1.9.

Acknowledgements This work has been partially supported by a grant P1.1B2012-47 of the Universidad Jaume I.

\section{References}

1. Brower, D., Clemence, G.M.: Celestial Mechanics. Academic, New York (1965)

2. Brumberg, E.V.: Length of arc as independent argument for highly eccentric orbits. Celest. Mech. 53, 323-328 (1992)

3. Fehlberg, E., Marsall, G.C.: Classical fifth, sixth, seventh and eighth Runge-Kutta formulas with stepsize control. Technical report, NASA, R-287 (1968)

4. Ferrándiz, J.M., Ferrer, S., Sein-Echaluce, M.L.: Generalized elliptic anomalies. Celest. Mech. 40, 315-328 (1987)

5. Gragg, W.B.: Repeated extrapolation to the limit in the numerical solution of ordinary differential equations. SIAM J. Numer. Anal. 2, 384-403 (1965)

6. Janin, G.: Accurate computation of highly eccentric satellite orbits. Celest. Mech. 10, 451-467 (1974)

7. Janin, G., Bond, V.R.: The elliptic anomaly. Technical memorandum, NASA, n. 58228 (1980)

8. Levallois, J.J., Kovalevsky, J.: Géodésie Générale, vol. 4. Eyrolles, Paris (1971)

9. López, J.A., Agost, V., Barreda, M.: A note on the use of the generalized Sundman transformations as temporal variables in celestial mechanics. Int. J. Comput. Math. 89, 433-442 (2012)

10. López, J.A., Marco, F.J., Martínez, M.J.: A study about the integration of the elliptical orbital motion based on a special one-parametric family of anomalies. Abstr. Appl. Anal. 2014, ID 162060, 1-11 (2014)

11. Nacozy, P.: The intermediate anomaly. Celest. Mech. 16, 309-313 (1977)

12. Sundman, K.: Memoire sur le probleme des trois corps. Acta Math. 36, 105-179 (1912)

13. Tisserand, F.F.: Traité de Mecanique Celeste. Gauthier-Villars, Paris (1896)

14. Velez, C.E., Hilinski, S.: Time transformation and Cowell's method. Celest. Mech. 17, 83-99 (1978) 